\section{Concentration and Timing of Ethephon Drench Applications Interact to Affect Growth and Flowering of Containerized Angelonia and Geranium}

\author{
Christopher J. Currey ${ }^{1,4}$, Kenneth G. McCabe $^{2}$, and Kellie J. Walters ${ }^{3}$ \\ Department of Horticulture, Iowa State University, 127 Horticulture Hall, \\ Ames, IA 50011
}

Additional index words. floriculture, greenhouse production, Pelargonium, plant growth retardant, summer snapdragon

\begin{abstract}
Ethephon drenches have been reported to effectively control growth of containerized bedding plants. However, previous researchers have indicated that the effects of ethephon drenches on growth and flowering may differ depending on the timing of applications. Our objectives were to quantify the effects of ethephon concentration, timing of substrate drench application, and their interaction on the growth, size, and flowering of two annual bedding plants. Angelonia (Angelonia angustifolia) and geranium (Pelargonium $\times$ hortorum), seedlings were planted in 10.2-cm-diameter containers filled with a commercial, soilless growing substrate composed of (by vol.) $75 \%$ sphagnum peatmoss, and $25 \%$ perlite. Five, 10,15 , or 20 days after transplanting seedlings, $70-\mathrm{mL}$ aliquots containing $0,50,100$, or $200 \mathrm{mg} \cdot \mathrm{L}^{-1}$ ethephon were applied as substrate drenches. Species varied in their growth and flowering responses to ethephon concentration, drench application timing, and their interaction. For angelonia, flowering was delayed most with early applications and high concentrations, and delay was diminished with later applications. Angelonia height was unaffected by late applications, though lateral growth was suppressed 20 days after transplant with $200 \mathrm{mg} \cdot \mathrm{L}^{-1}$ ethephon. Flowering of geranium was only delayed when ethephon was applied 5 days after transplanting, whereas flowering, vegetative height, and shoot dry weight were affected more by earlier applications and higher concentrations. Width and root weight were only affected by ethephon concentration, with growth suppression increasing as concentrations increased. Ethephon is an effective growth regulator when applied as a substrate drench. However, the degree of activity and resulting impact on flowering, size, and growth is influenced by the interaction between ethephon concentrations and the timing of drench applications after transplanting.
\end{abstract}

In 2015 , the wholesale value of floriculture crops for the top 15 producing states was $\$ 4.37$ billion USD (U.S. Department of Agriculture, 2016). Of that total value, annual bedding and garden plants were the most valuable sector, valued at $\$ 1.29$ billion USD. Controlling growth of containerized annuals is desirable for several reasons, including producing a plant that is proportionately sized for containers (Vernieri et al., 2003), as well as increasing planting densities during greenhouse production (Carey et al., 2007) and

Received for publication 2 Aug. 2016. Accepted for publication 3 Oct. 2016

We gratefully acknowledge Peter Lawlor for greenhouse assistance, Nicholas Flax and Alexander Litvin for thoughtful critique of the manuscript, Wagner Greenhouses for plant material, and Fine Americas for the plant growth regulator.

The use of trade names in this publication does not imply endorsement by Iowa State University of products named nor criticism of similar ones not mentioned.

${ }^{1}$ Assistant professor

${ }^{2}$ Research associate and former graduate student. ${ }^{3}$ Former graduate student.

${ }^{4}$ Corresponding author. E-mail: ccurrey@iastate.edu. inhibits conversion of biologically inactive forms of gibberellic acid into active forms. Cell elongation is suppressed as a result of gibberellic acid biosynthesis inhibition and, therefore, stem elongation and plant size can be controlled. Although ethephon is a commonly used PGR, its mode of action differs compared with other PGR active ingredients. Instead of affecting gibberellic acid synthesis, ethephon breaks down inside plant cells and generates ethylene (Barrett, 2001). In addition to diminishing apical dominance, suppressing flower development and inducing flower abortion, and promoting fruit ripening, increased ethylene concentrations causes microfibril orientation in cell walls to change and inhibits latitudinal cell elongation, which diminishes stem elongation and, therefore, plant size (Burg, 1973).

The majority of PGR applications are administered using either foliar sprays or substrate drenches (Gent and McAvoy, 2000). Spray applications are easy to apply and can provide adequate growth control, though substrate drenches can increase growth control uniformity and duration (Currey et al., 2010; Currey and Lopez, 2011; Currey et al., 2012). Ethephon is not currently labeled for application via substrate drenches, though recent research reports the promise of using drenches for growth control (Aiken et al., 2015; Miller et al., 2012). Miller et al. (2012) noted that ethephon drench activity appeared to vary when plants were treated early ( $2 \mathrm{~d}$ after transplanting) as opposed to later (10 d after transplanting); however, application times were not a controlled factor and varied across locations. Since PGR application timing is important to elicit desirable and appropriate plant responses, it is important to quantify the effect of application timing to determine best management practices for using ethephon drenches (Bailey and Whipker, 1998). The objectives of our study were to quantify the effect of ethephon drench concentration and application time after transplanting on growth and development of containerized flowering annuals.

\section{Materials and Methods}

(Alem et al., 2015), and minera nutrition (Haver and Schuch, 1996) can be manipulated to control growth. However, it can be difficult to employ these techniques when a wide range of species are produced in a single greenhouse, as is commonly the case during spring annual production. Chemical plant growth retardants (PGRs) are frequently used to suppress stem elongation and targeted, species-specific applications can be made and are useful in controlling annual bedding plant growth (Dole and Wilkins, 2005; Whipker et al., 2011).

The mode of action for the majority of PGRs is inhibiting specific steps of the gibberellic acid biosynthesis pathway (Rademacher, 2000). Chlormequat chloride inhibits the conversion of geranylgeranyl pyrophosphate to copalyl diphosphate and, to a lesser extent, ent-kaurene synthesis. Ancymidol, flurprimidol, paclobutrazol, and uniconazole are all closely related compounds which inhibit synthesis of ent-kaurenoic acid, whereas daminozide
'Serena White' angelonia and 'Pinto Premium Deep Red' geranium grown in 288-cell trays were received from a commercial plug producer (Wagner Greenhouses, Minneapolis, MN). Seedlings were individually planted in 10.2-cm-diameter containers $(490 \mathrm{~mL}$ vol.; $\mathrm{HC}$ Companies, Middlefield, $\mathrm{OH}$ ) filled with a commercial soilless substrate composed of (by vol.) $75 \%$ canadian sphagnum peatmoss and $25 \%$ perlite and amended with dolomitic limestone, starter nutrient charge, and a surfactant (Sunshine Mix No. 1; Sun Gro Horticulture, Agawam, MA).

Plants were grown on expanded metal benches in a glass-glazed greenhouse at Iowa State University, Ames, IA (lat. $42^{\circ} \mathrm{N}$ ) with fog cooling, radiant hot-water floor and perimeter heating, and retractable shade curtains controlled by an environmental computer (ARGUS Titan, ARGUS Control Systems, Surrey, British Columbia, Canada). The day 
and night greenhouse air temperature set points were $22.5 \pm 1$ and $18.0 \pm 1{ }^{\circ} \mathrm{C}$, respectively. High-pressure sodium lamps delivered a supplemental photosynthetic photon flux of $\approx 190 \mu \mathrm{mol} \cdot \mathrm{m}^{-2} \cdot \mathrm{s}^{-1}$ at plant height [as measured with a quantum sensor (LI-190 SB; LI-COR Biosciences, Lincoln, NE)] when ambient light intensity was below $100 \mu \mathrm{mol} \cdot \mathrm{m}^{-2} \cdot \mathrm{s}^{-1}$ between 0600 and 2200. Plants were irrigated with tap water supplemented with a blend of water-soluble fertilizers $\left(50\right.$ and $100 \mathrm{mg} \cdot \mathrm{L}^{-1} \mathrm{~N}$ provided from $21 \mathrm{~N}-2.2 \mathrm{P}-16.6 \mathrm{~K}$ and $15 \mathrm{~N}-$ $2.2 \mathrm{P}-12.5 \mathrm{~K}$, respectively; Everris NA, Inc., Marysville, $\mathrm{OH})$ to provide the following (in $\mathrm{mg} \cdot \mathrm{L}^{-1}$ ): 150 nitrogen, 20 phosphorous, 123 potassium, 33 calcium, 13 magnesium, 0.5 iron, 0.4 manganese and zinc, 0.2 copper and boron, and 0.08 molybdenum.

Five days after transplanting seedlings, PGR drench treatments were initiated. Each plant was provided with a $70-\mathrm{mL}$ aliquot of solution containing deionized water and 0 , 50, 100 , or $200 \mathrm{mg} \cdot \mathrm{L}^{-1}$ ethephon (Collate; Fine Americas, Inc., Walnut Creek, CA) 5, 10,15 , or $20 \mathrm{~d}$ after transplanting.

The date the first flower opened was recorded for each plant during the experiment. Angelonia and geranium growth data were collected 7 and 8 weeks after planting, respectively. Plant height from the surface of the substrate to the tallest growing point (angelonia and geranium) and top of the leaf canopy (geranium only) and the widths at the widest point and $90^{\circ}$ from the widest point were recorded. Shoots, severed at the surface of the substrate, and roots, with the substrate gently washed from roots, were placed in separate paper bags and placed in a forced-air oven maintained at $67^{\circ} \mathrm{C}$ for $3 \mathrm{~d}$, after which they were weighed and dry weight was recorded.

The experimental design was a completely randomized design in factorial arrangement for each species, with ethephon concentration (four levels) and application time (four levels) as factors. There were 10 replicates (individual plants) for each ethephon concentration and application time combination. Analyses of variance and mean separation using Tukey's honestly significant difference test at $P \leq 0.05$ were performed using SPSS 21.0 (IBM Corporation, Armonk, NY).

\section{Results}

Angelonia. Flowering of angelonia was affected by the interactions of ethephon drench concentration and application time (Table 1). Plants treated with $0 \mathrm{mg} \cdot \mathrm{L}^{-1}$ ethephon flowered in 34.9-35.7 $\mathrm{d}$ across application times. Alternatively, for plants treated with $50-200 \mathrm{mg} \cdot \mathrm{L}^{-1}$ ethephon, flowering delay decreased with later application times, but increased with concentration. When 50 or $200 \mathrm{mg} \cdot \mathrm{L}^{-1}$ was applied $5 \mathrm{~d}$ after transplanting, flowering was delayed by 4.2 or $7.7 \mathrm{~d}$, respectively, compared with control plants. For plants treated $20 \mathrm{~d}$ after transplanting flowering was only delayed by 2.0 or $2.2 \mathrm{~d}$ compared with control plants when treated with 100 or $200 \mathrm{mg} \cdot \mathrm{L}^{-1}$ ethephon.

Concentration and application also interacted to affect the height and width of angelonia

Table 1. Time to flower, flowering height, canopy height, and shoot dry weight of angelonia (Angelonia angustifolia 'Serena White') treated with ethephon drenches. Seedlings were planted into 10.2-cmdiameter round containers filled with a commercial soilless substrate composed of (by vol.) $75 \%$ canadian sphagnum and $25 \%$ peatmoss and perlite and provided with $70-\mathrm{mL}$ aliquots containing 0,50 , 100 , or $200 \mathrm{mg} \cdot \mathrm{L}^{-1}$ ethephon applied $5,10,15$, or $20 \mathrm{~d}$ after transplanting seedlings.

\begin{tabular}{|c|c|c|c|c|}
\hline \multirow[b]{2}{*}{ Ethephon concn $\left(\mathrm{mg} \cdot \mathrm{L}^{-1}\right)$} & \multicolumn{4}{|c|}{ Application time (d after transplanting) } \\
\hline & 5 & 10 & 15 & 20 \\
\hline \multicolumn{5}{|c|}{ Time to flower (d) } \\
\hline 0 & $35.3 \mathrm{c}^{\mathrm{z}} \mathrm{A}^{\mathrm{y}}$ & $35.6 \mathrm{bA}$ & $35.7 \mathrm{cA}$ & $34.9 \mathrm{bA}$ \\
\hline 50 & 39.5 bA & $38.4 \mathrm{aAB}$ & $36.4 \mathrm{bcBC}$ & $35.4 \mathrm{abC}$ \\
\hline 100 & $40.7 \mathrm{abA}$ & $38.5 \mathrm{aAB}$ & $38.1 \mathrm{abB}$ & $36.9 \mathrm{aB}$ \\
\hline 200 & $43.0 \mathrm{aA}$ & $40.3 \mathrm{aAB}$ & $39.2 \mathrm{aBC}$ & $37.1 \mathrm{aC}$ \\
\hline Concn $(\mathrm{C})$ & $* * *$ & & & \\
\hline Time $(\mathrm{T})$ & $* * *$ & & & \\
\hline $\mathrm{C} \times \mathrm{T}$ & $* * *$ & & & \\
\hline \multicolumn{5}{|c|}{ Height $(\mathrm{cm})$} \\
\hline 0 & $31.4 \mathrm{aA}$ & $30.2 \mathrm{aA}$ & $30.5 \mathrm{aA}$ & $30.5 \mathrm{aA}$ \\
\hline 50 & $22.0 \mathrm{bB}$ & $28.0 \mathrm{abA}$ & $30.3 \mathrm{aA}$ & $30.3 \mathrm{aA}$ \\
\hline 100 & $21.2 \mathrm{bcB}$ & $26.0 \mathrm{bcA}$ & $27.9 \mathrm{aA}$ & $28.5 \mathrm{aA}$ \\
\hline 200 & $19.2 \mathrm{cC}$ & $24.8 \mathrm{cB}$ & $27.7 \mathrm{aA}$ & $28.4 \mathrm{aA}$ \\
\hline $\mathrm{C}$ & $* * *$ & & & \\
\hline $\mathrm{T}$ & $* * *$ & & & \\
\hline $\mathrm{C} \times \mathrm{T}$ & $* * *$ & & & \\
\hline \multicolumn{5}{|c|}{ Width $(\mathrm{cm})$} \\
\hline 0 & $27.2 \mathrm{aA}$ & $27.2 \mathrm{aA}$ & $28.1 \mathrm{aA}$ & $27.0 \mathrm{aA}$ \\
\hline 50 & $14.4 \mathrm{bC}$ & $21.3 \mathrm{bB}$ & $24.3 \mathrm{bAB}$ & $25.4 \mathrm{abA}$ \\
\hline 100 & $12.3 \mathrm{bcC}$ & $17.0 \mathrm{cB}$ & $21.0 \mathrm{bcA}$ & $23.3 \mathrm{abA}$ \\
\hline 200 & $11.3 \mathrm{cC}$ & $16.1 \mathrm{cB}$ & $18.3 \mathrm{cB}$ & $21.9 \mathrm{bA}$ \\
\hline $\mathrm{C}$ & $* * *$ & & & \\
\hline $\mathrm{T}$ & $* * *$ & & & \\
\hline $\mathrm{C} \times \mathrm{T}$ & $* * *$ & & & \\
\hline \multicolumn{5}{|c|}{ Shoot dry weight (g) } \\
\hline 0 & $5.2 \mathrm{aA}$ & $5.0 \mathrm{aA}$ & $5.6 \mathrm{aA}$ & $5.3 \mathrm{aA}$ \\
\hline 50 & $2.1 \mathrm{bC}$ & $4.1 \mathrm{bB}$ & $5.1 \mathrm{abA}$ & $5.2 \mathrm{aA}$ \\
\hline 100 & $1.8 \mathrm{bC}$ & $3.3 \mathrm{cB}$ & $4.5 \mathrm{bcA}$ & $4.6 \mathrm{bA}$ \\
\hline 200 & $1.7 \mathrm{bD}$ & $3.0 \mathrm{cC}$ & $3.9 \mathrm{cB}$ & $4.6 \mathrm{bA}$ \\
\hline $\mathrm{C}$ & $* * *$ & & & \\
\hline $\mathrm{T}$ & $* * *$ & & & \\
\hline $\mathrm{C} \times \mathrm{T}$ & *** & & & \\
\hline \multicolumn{5}{|c|}{ Root dry weight (g) } \\
\hline 0 & $1.06 \mathrm{aA}$ & $0.94 \mathrm{aA}$ & $1.04 \mathrm{aA}$ & $0.96 \mathrm{aA}$ \\
\hline 50 & $0.33 \mathrm{bC}$ & $0.69 \mathrm{bB}$ & $0.76 \mathrm{bAB}$ & $0.88 \mathrm{abA}$ \\
\hline 100 & $0.29 \mathrm{bcC}$ & $0.57 \mathrm{bB}$ & $0.66 \mathrm{bcB}$ & $0.79 \mathrm{bcA}$ \\
\hline 200 & $0.23 \mathrm{cD}$ & $0.43 \mathrm{cC}$ & $0.57 \mathrm{cB}$ & $0.73 \mathrm{cA}$ \\
\hline $\mathrm{C}$ & $* * *$ & & & \\
\hline $\mathrm{T}$ & $* * *$ & & & \\
\hline $\mathrm{C} \times \mathrm{T}$ & $* * *$ & & & \\
\hline
\end{tabular}

${ }^{\mathrm{z}}$ Within-column means followed by different lower case letters are significantly different by Tukey's honestly significant difference () test at $P \leq 0.05$.

${ }^{y}$ Within-row means followed by different upper case letters are significantly different by Tukey's test at $P \leq 0.05$.

***Indicates significant at $P \leq 0.001$.

(Table 1). Plants were $30.2-31.4 \mathrm{~cm}$ tall across application times when no ethephon was applied. When angelonia were treated $5 \mathrm{~d}$ after transplanting, plant height was 9.4 to $12.2 \mathrm{~cm}$ shorter than untreated plants as ethephon concentration increased from 50 to $200 \mathrm{mg} \cdot \mathrm{L}^{-1}$. There were no differences in height for plants untreated or treated with ethephon 10 or $20 \mathrm{~d}$ after transplanting.

Angelonia width was $27.0-28.1 \mathrm{~cm}$ for untreated plants across application times. For early applications (5 $\mathrm{d}$ after transplanting) widths were less with increasing ethephon concentrations, from $14.4 \mathrm{~cm}\left(50 \mathrm{mg} \cdot \mathrm{L}^{-1}\right)$ to $11.3 \mathrm{~cm}\left(200 \mathrm{mg} \cdot \mathrm{L}^{-1}\right)$. For the latest application ( $20 \mathrm{~d}$ after transplanting), width of plants treated with $200 \mathrm{mg} \cdot \mathrm{L}^{-1}$ was $5.1 \mathrm{~cm}$ less than untreated plants.

Concentration, application time, and their interaction affected shoot and root dry weight of angelonia (Table 1). The shoot weight of the untreated controls ranged from 5.0 to $5.6 \mathrm{~g}$. Shoot dry weight was lower than untreated plants with increasing ethephon concentrations, but the effect diminished with later applications. For example, shoot weight was 3.1-3.5 g less than untreated plants treated when treated $5 \mathrm{~d}$ after transplanting. Alternatively, when 100 or $200 \mathrm{mg} \cdot \mathrm{L}^{-1}$ ethephon was applied $20 \mathrm{~d}$ after transplanting, shoot dry weight was $0.7 \mathrm{~g}$ less than untreated plants.

Root dry weight was $0.94-1.06 \mathrm{~g}$ across application times for plants treated with $0 \mathrm{mg} \cdot \mathrm{L}^{-1}$ ethephon. When ethephon was applied $5 \mathrm{~d}$ after transplanting, root weight was $0.73-0.83 \mathrm{~g}$ less for plants treated with $50-200 \mathrm{mg} \cdot \mathrm{L}^{-1}$ ethephon compared with control plants; alternatively, root weight was 0.17 or $0.23 \mathrm{~g}$ less than untreated plants when applications were made $20 \mathrm{~d}$ after transplanting.

Geranium. Ethephon drench concentration and application time interacted to affect 
geranium flowering (Table 2). Plants treated with $0 \mathrm{mg} \cdot \mathrm{L}^{-1}$ ethephon flowered $46.7-48.7 \mathrm{~d}$ after transplanting, across application time. $5 \mathrm{~d}$ after transplanting, flowering was delayed by $17.0-19.3 \mathrm{~d}$ compared with untreated plants. Alternatively, there were no differences in time to flower across ethephon concentrations when drenches were applied $10 \mathrm{~d}$ or more after transplanting.

Flowering heights of untreated geranium plants were 29.0-29.6 cm (Table 2). Although all ethephon drenches suppressed pedicle elongation regardless of application time, the magnitude of effect was greater for earlier applications. For example, flowering height was 6.8 to $8.9 \mathrm{~cm}$ shorter than untreated plants, respectively, as concentration increased from 50 to $200 \mathrm{mg} \cdot \mathrm{L}^{-1}$ when applied $5 \mathrm{~d}$ after transplanting, or 3.3 to $5.6 \mathrm{~cm}$ shorter, respectively, when treated $20 \mathrm{~d}$ after transplanting.

For applications made between 5 and $15 \mathrm{~d}$ after transplanting, only plants treated with 100 or $200 \mathrm{mg} \cdot \mathrm{L}^{-1}$ had shorter canopy heights, whereas plants treated $20 \mathrm{~d}$ after transplanting were 3.1$3.2 \mathrm{~cm}$ shorter than untreated plants (Table 2).

Width was affected by ethephon concentration and not application time nor their When 50-200 mg. $\mathrm{L}^{-1}$ ethephon was applied

interaction (Table 3). As ethephon concentrations increased from 50 to $200 \mathrm{mg} \cdot \mathrm{L}^{-1}$, suppression of plant width increased from 1.0 to $1.5 \mathrm{~cm}$.

Ethephon concentration and application time interacted to affect shoot dry weight (Table 2). For plants treated with $0 \mathrm{mg} \cdot \mathrm{L}^{-1}$ ethephon drenches, shoot dry weight was $7.4-8.0 \mathrm{~g}$. When drenches were applied $5 \mathrm{~d}$ after transplanting, shoot dry weight was 1.0 to $1.9 \mathrm{~g}$ less than untreated plants as ethephon concentration increased from 50 to $200 \mathrm{mg} \cdot \mathrm{L}^{-1}$. Alternatively, when drenches were applied $20 \mathrm{~d}$ after transplanting, shoot dry weight was only impacted by plants treated with $200 \mathrm{mg} \cdot \mathrm{L}^{-1}$ and was $0.9 \mathrm{~g}$ less than untreated plants.

Root dry weight was unaffected by ethephon concentration, but not application time or the interaction of ethephon concentration and application time (Table 3). Root weight for plants treated with $0 \mathrm{mg} \cdot \mathrm{L}^{-1}$ was $0.85 \mathrm{~g}$, but was $0.76 \mathrm{~g}$ as ethephon concentration increased to $200 \mathrm{mg} \cdot \mathrm{L}^{-1}$.

\section{Discussion}

We found that ethephon substrate drenches delayed flowering of angelonia and geranium.

Table 2. Time to flower, flowering height, canopy height, and shoot dry weight of geranium (Pelargonium $\times$ hortorum 'Pinto Premium Deep Red') treated with ethephon drenches. Seedlings were planted into 10.2-cm-diameter round containers filled with a commercial soilless substrate composed of (by vol.) $75 \%$ canadian sphagnum peatmoss and $25 \%$ perlite and provided with $70-\mathrm{mL}$ aliquots containing 0,50 , 100 , or $200 \mathrm{mg} \cdot \mathrm{L}^{-1}$ ethephon applied $5,10,15$, or $20 \mathrm{~d}$ after transplanting seedlings.

\begin{tabular}{|c|c|c|c|c|}
\hline \multirow[b]{2}{*}{ Ethephon concn $\left(\mathrm{mg} \cdot \mathrm{L}^{-1}\right)$} & \multicolumn{4}{|c|}{ Application time (d after transplanting) } \\
\hline & 5 & 10 & 15 & 20 \\
\hline \multicolumn{5}{|c|}{ Time to flower (d) } \\
\hline 0 & $48.7 \mathrm{~b}^{\mathrm{z}} \mathrm{A}^{\mathrm{y}}$ & $47.6 \mathrm{aA}$ & $46.7 \mathrm{aA}$ & $47.7 \mathrm{aA}$ \\
\hline 50 & $65.7 \mathrm{aA}$ & $51.1 \mathrm{aB}$ & $47.6 \mathrm{aB}$ & $48.0 \mathrm{aB}$ \\
\hline 100 & $68.0 \mathrm{aA}$ & $52.4 \mathrm{aB}$ & $48.0 \mathrm{aB}$ & $48.3 \mathrm{aB}$ \\
\hline 200 & $68.0 \mathrm{aA}$ & $52.6 \mathrm{aB}$ & $48.2 \mathrm{aB}$ & $48.5 \mathrm{aB}$ \\
\hline Concn (C) & $* * *$ & & & \\
\hline Time $(\mathrm{T})$ & $* * *$ & & & \\
\hline $\mathrm{C} \times \mathrm{T}$ & $* * *$ & & & \\
\hline \multicolumn{5}{|c|}{ Flowering height $(\mathrm{cm})$} \\
\hline 0 & $29.2 \mathrm{aA}$ & $29.6 \mathrm{aA}$ & $29.3 \mathrm{aA}$ & $29.0 \mathrm{aA}$ \\
\hline 50 & $22.4 \mathrm{bB}$ & $25.9 \mathrm{bA}$ & $26.2 \mathrm{bA}$ & $25.7 \mathrm{bA}$ \\
\hline 100 & $23.1 \mathrm{bA}$ & $23.6 \mathrm{bA}$ & $24.5 \mathrm{bA}$ & $24.5 \mathrm{bcA}$ \\
\hline 200 & $20.3 \mathrm{cB}$ & $20.1 \mathrm{~dB}$ & $22.1 \mathrm{cA}$ & $23.4 \mathrm{cA}$ \\
\hline $\mathrm{C}$ & $* * *$ & & & \\
\hline $\mathrm{T}$ & $* * *$ & & & \\
\hline $\mathrm{C} \times \mathrm{T}$ & $* * *$ & & & \\
\hline \multicolumn{5}{|c|}{ Canopy height $(\mathrm{cm})$} \\
\hline 0 & $19.5 \mathrm{aA}$ & $19.2 \mathrm{aA}$ & $18.8 \mathrm{aA}$ & $19.8 \mathrm{aA}$ \\
\hline 50 & $17.1 \mathrm{abAB}$ & $18.0 \mathrm{abA}$ & $18.0 \mathrm{aA}$ & $16.7 \mathrm{bB}$ \\
\hline 100 & $18.0 \mathrm{bA}$ & $16.5 \mathrm{bB}$ & $16.1 \mathrm{bB}$ & $16.8 \mathrm{bB}$ \\
\hline 200 & $16.6 \mathrm{cA}$ & $16.5 \mathrm{bA}$ & $16.1 \mathrm{bA}$ & $16.6 \mathrm{bA}$ \\
\hline $\mathrm{C}$ & $* * *$ & & & \\
\hline $\mathrm{T}$ & NS & & & \\
\hline $\mathrm{C} \times \mathrm{T}$ & $* * *$ & & & \\
\hline \multicolumn{5}{|c|}{ Shoot dry weight (g) } \\
\hline 0 & $7.5 \mathrm{aA}$ & $7.5 \mathrm{aA}$ & $7.4 \mathrm{aA}$ & $8.0 \mathrm{aA}$ \\
\hline 50 & $6.5 \mathrm{bB}$ & $7.4 \mathrm{bA}$ & $7.6 \mathrm{aA}$ & $7.6 \mathrm{abA}$ \\
\hline 100 & $6.8 \mathrm{bC}$ & $6.9 \mathrm{abBC}$ & $7.5 \mathrm{aAB}$ & $7.9 \mathrm{aA}$ \\
\hline 200 & $5.7 \mathrm{cC}$ & $6.5 \mathrm{bB}$ & $6.7 \mathrm{bAB}$ & $7.1 \mathrm{bA}$ \\
\hline $\mathrm{C}$ & $* * *$ & & & \\
\hline $\mathrm{T}$ & $* * *$ & & & \\
\hline $\mathrm{C} \times \mathrm{T}$ & $*$ & & & \\
\hline
\end{tabular}

${ }^{\mathrm{z}}$ Within-column means followed by different lower case letters are significantly different by Tukey's honestly significant difference (HSD) test at $P \leq 0.05$.

${ }^{\mathrm{y}}$ Within-row means followed by different upper case letters are significantly different by Tukey's HSD test at $P \leq 0.05$.

Ns, *, ${ }^{* * *}$ Nonsignificant or significant at $P \leq 0.05$ or 0.001 , respectively.
Our results agree with previous reports on the effects of ethephon drenches on annuals (Aiken et al., 2015; Miller et al., 2012). Miller et al. (2012) reported time to flower for numerous annuals treated with ethephon at different institutions. Plants were treated $2 \mathrm{~d}$ after transplanting at Cornell University and, while flowering for snapdragon (Antirrhinum majus) and dianthus (Dianthus chinensis) were unaffected by ethephon, flowering of eight other species was delayed by 1.8-30.7 $\mathrm{d}$. When plants at Michigan State University were treated $10 \mathrm{~d}$ after transplanting, tomato (Lycopersicon esculentum) and petunia flowering was delayed, whereas snapdragon, bedding impatiens (Impatiens walleriana), and osteospermum (Osteospermum fruticosum) flowering was not. Interestingly, the same osteospermum cultivar (Zion Copper Amethyst) and cell size was used at both Cornell and Michigan State and, whereas flowering was delayed for plants treated $2 \mathrm{~d}$ after transplanting at Cornell, time to flower was unaffected by ethephon applied $10 \mathrm{~d}$ after transplanting at Michigan State. Aiken et al. (2015) reported that while veronica (Veronica spicata) flowering was unaffected by ethephon drenches, verbena (Verbena bonariensis) flowered up to $\approx 2$ weeks later than untreated plants; though flowering delays were greater when substrate $\mathrm{pH}$ was more acidic.

Ethephon is used to purposefully delay flowering for different types of crops. For example, stock plant producers may use ethephon to maintain stock plants and keep cuttings in a vegetative state (Glady et al., 2007; Hayashi et al., 2001). Similarly, cuttings can be treated with ethephon during propagation to further inhibit premature flowering (Runkle, 2013). After transplanting, plants may be treated with ethephon to prevent premature flowering during vegetative growth or the "bulking up" phase, or when production times are long (Currey and Flax, 2015). Using ethephon to control flowering is primarily for crops that are already mature and flower readily, such as

Table 3. Width and root dry weight of geranium (Pelargonium $\times$ hortorum 'Pinto Premium Deep Red') treated with ethephon drenches. Seedlings were planted into $10.2-\mathrm{cm}$-diameter round containers filled with a commercial soilless substrate composed of (by vol.) 75\% canadian sphagnum peatmoss and $25 \%$ perlite and provided with $70-\mathrm{mL}$ aliquots containing $0,50,100$, or $200 \mathrm{mg} \cdot \mathrm{L}^{-1}$ ethephon. Since neither application time nor the interaction of concentration with application time were significant, data across application time were pooled.

\begin{tabular}{lcc}
\hline $\begin{array}{l}\text { Ethephon } \\
\text { concn }\left(\mathrm{mg} \cdot \mathrm{L}^{-1}\right)\end{array}$ & Width $(\mathrm{cm})$ & Root weight $(\mathrm{g})$ \\
\hline 0 & $22.8 \mathrm{a}^{\mathrm{z}}$ & $0.85 \mathrm{a}$ \\
50 & $21.8 \mathrm{bc}$ & $0.85 \mathrm{a}$ \\
100 & $22.1 \mathrm{~b}$ & $0.83 \mathrm{ab}$ \\
200 & $21.3 \mathrm{c}$ & $0.76 \mathrm{~b}$ \\
Concn $(\mathrm{C})$ & $* * *$ & $* * *$ \\
Time $(\mathrm{T})$ & NS & NS \\
$\mathrm{C} \times \mathrm{T}$ & NS & NS \\
\hline
\end{tabular}

${ }^{\mathrm{z}}$ Within-column means followed by different lower case letters are significantly different by Tukey's honestly significant difference test at $P \leq 0.05$. Ns, **** Nonsignificant or significant at $P \leq 0.05$. 
vegetatively propagated crops, or for production in larger containers where plants begin to flower before they are proportionally sized. Production cycles are relatively short and flowering delays increase crop time for seed-propagated annual bedding plants grown in containers. Although recommendations for foliar ethephon spray applications are to cease 6 weeks before marketing to allow flowers to develop for sales and marketing, we observed an opposite trend in our research with the earliest drenches impacting flowering the most and later applications having minimal (angelonia) or no effect (geranium) on time to flower (Tables 1 and 2). The effect of ethephon drenches and sprays on flowering warrants further study to elucidate and better understand how these application methods impact flowering differently. Substrate drenches may serve as a preferred ethephon application method for short-term crops when controlling size, but not flowering, is desired.

In addition to flowering, ethephon drenches controlled size (heights and widths) and growth (dry weight) of angelonia and geranium. This work agrees with previous research on ethephon drenches which reported successful growth control of containerized annuals and perennials (Aiken et al., 2015; Miller et al., 2012). Aiken et al. (2015) reported that veronica treated with ethephon drenches were narrower than untreated plants with no differences in plant height, whereas height and width of treated verbena were consistently smaller than untreated plants. Variation in plant responses to ethephon across study locations was reported by Miller et al. (2012). Although height at flowering for snapdragon or bedding impatiens was suppressed compared with untreated plants, ethephon had no effect on osteospermum or petunia height at Michigan State University. At Cornell University, although ethephon drenches did not affect lobelia ( $\mathrm{LO}$ belia erinus), 10 other bedding plants were $18 \%$ to $70 \%$ shorter than untreated plants when treated with ethephon. It is clear that bedding plant growth can be controlled when our results on ethephon drenches are taken together with previous studies. However, there are species-specific responses to ethephon drenches that warrant broad studies screening various containerized bedding plants for responses to ethephon drenches.

In addition to size, ethephon drenches also diminished angelonia and geranium shoot and root dry weight. Shoot dry weight of verbena and veronica treated with ethephon drenches was less compared with treated plants across a range of substrate $\mathrm{pH}$ (Aiken et al., 2015). Miller et al. (2012) reported shoot and root weights were affected by ethephon drenches differently across locations. Shoot and root dry weight of annuals treated at Cornell University were $12 \%$ to $81 \%$ and $2 \%$ to $85 \%$ less, respectively, than untreated plants. Alternatively, when ethephon was applied $10 \mathrm{~d}$ after transplanting to bedding plants at Purdue University, shoot and root weight were $5 \%$ to $61 \%$ and $4 \%$ to $73 \%$ smaller, respectively, compared with untreated plants. Though shoot and root weight were affected by ethephon drenches at both locations, growth suppression was greater for plants treated earlier (Cornell University) as opposed to those treated later (Purdue University). Less shoot weight can be acceptable as long as plants are still considered marketable with respect to size and quality. Since containerized bedding plants are transplanted to large containers such as hanging baskets or patio containers or into beds, care should be taken to minimize excessive suppression of root growth which may negatively impact transplant establishment and subsequent performance. Extremely, early application (i.e., $5 \mathrm{~d}$ after transplanting) of ethephon drenches should be avoided for this reason.

Although we have found no research systematically evaluating the effect of ethephon drench application time after transplanting, we have found previous reports of ethephon drench applications to bulb crops at different physiological stages (Moe, 1980; Wees, 1993). Moe (1980) reported suppression of daffodil (Narcissus pseudonarcissus) peduncle and leaf elongation increased with ethephon concentration, but decreased with later applications. For example, when daffodil were treated with ethephon when leaves were $6 \mathrm{~cm}$ long, flowers and leaves were $12 \%$ to $29 \%$ and $11 \%$ to $18 \%$ shorter, respectively, compared with untreated plants. However, when ethephon was applied when leaves were $18 \mathrm{~cm}$ long, daffodil flowers and leaves were $9 \%$ to $24 \%$ and $9 \%$ to $17 \%$ shorter, respectively, than untreated plants. Similarly, Wees (1993) treated 'Nellie White' easter lilies (Lilium longiflorum) when shoots were $10-16 \mathrm{~cm}$ tall (1989 or 1990 season) or 7$9 \mathrm{~cm}$ tall (1990 or 1991 season). Easter lilies were $17 \%$ to $53 \%$ shorter than untreated plants when taller shoots were treated, whereas lilies were $36 \%$ to $67 \%$ shorter than untreated plants when shorter shoots were treated. The results from these studies and our research support the hypothesis that ethephon drench application timing affects the magnitude of growth inhibition, whereby early applications have a greater growth-suppressing effect compared with later applications. However, the stronger activity associated with earlier application times can be excessive, with respect to the degree of growth control.

\section{Conclusions}

When the effects of ethephon concentration, application timing, and their interaction on flowering, size, and growth are taken together for both angelonia and geranium and from previous research, we may draw some conclusions for drench applications to herbaceous annuals. We believe that applying ethephon drenches $\leq 5 \mathrm{~d}$ after transplanting should not be used for growth control in commercial production. For angelonia, flowering delays were greatest and commercially significant (i.e., $>7 \mathrm{~d}$ ) with early applications, whereas control of size and growth was excessive and resulted in unmarketable plants (Fig. 1). Though the size and growth of geraniums treated $5 \mathrm{~d}$ after transplanting resulted in appropriately sized plants for marketing, the flowering delay of $>2$ weeks is unacceptable for commercial production (Fig. 2). For applications made between 10 and $20 \mathrm{~d}$ after transplanting, concentration selection should depend on the degree of activity desired. Within a given application time, increasing the concentration will increase the magnitude of effect, so greater growth control may be achieved by simply increasing concentrations. We believe that when trying to achieve a similar degree of growth control across application times, ethephon concentration should increase with application time. For example, a lower concentration of $50 \mathrm{mg} \cdot \mathrm{L}^{-1}$ may produce appropriate control for earlier applications ( $10 \mathrm{~d}$ after transplanting), whereas a higher concentration of $200 \mathrm{mg} \cdot \mathrm{L}^{-1}$ may produce acceptable control for later applications (20 d after transplanting).

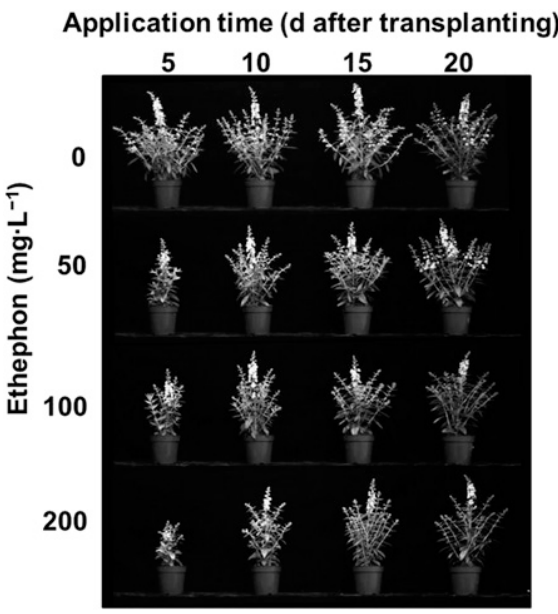

Fig. 1. Angelonia (Angelonia angustifolia 'Serena White') grown in 10.2-cm-diameter containers filled with a commercial soilless substrate composed of (by vol.) $75 \%$ canadian sphagnum peatmoss and $25 \%$ perlite and provided with $70-\mathrm{mL}$ aliquots containing $0,50,100$, or 200 $\mathrm{mg} \cdot \mathrm{L}^{-1}$ ethephon. Photos taken 6 weeks after transplanting

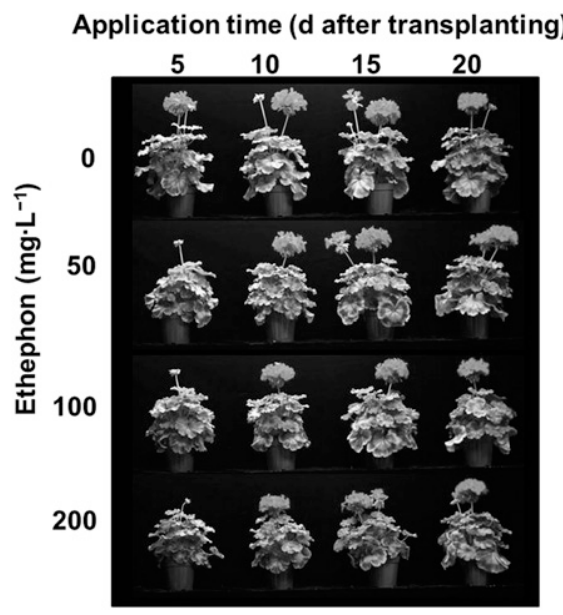

Fig. 2. Geraniums (Pelargonium $\times$ hortorum 'Pinto Premium Deep Red') grown in 10.2-cmdiameter containers filled with a commercial soilless substrate composed of (by vol.) $75 \%$ canadian sphagnum peatmoss and $25 \%$ perlite and provided with $70-\mathrm{mL}$ aliquots containing 0 , 50,100 , or $200 \mathrm{mg} \cdot \mathrm{L}^{-1}$ ethephon. Photos taken 7 weeks after transplanting. 


\section{Literature Cited}

Aiken, M.G., H.L. Scoggins, and J.G. Latimer. 2015. Substrate $\mathrm{pH}$ impacts efficacy of ethephon drenches on growth of herbaceous perennials. HortScience 50:1187-1191.

Alem, P., P.A. Thomas, and M.W. van Iersel. 2015. Controlled water deficit as an alternative to plant growth regulation of poinsettia stem elongation. HortScience 50:565-569.

Bailey, D. and B. Whipker. 1998. Best management practices for plant growth regulators used in floriculture. North Carolina State Univ. Hort. Info. Lflt. 529.

Barrett, J. 2001. Mechanisms of action, p. 32-41. In: M.L. Gaston, P.S. Konjoian, L.A. Kunkle, and M.F. Wilt (eds.). Tips on regulating growth of floriculture crops. OFA Services, Columbus, $\mathrm{OH}$.

Burg, S.P. 1973. Ethylene in plant growth. Proc. Natl. Acad. Sci. USA 70:591-597.

Carey, D., B. Whipker, I. McCall, and W. Buhler. 2007. Cytokinin based PGR affects growth of vegetative petunia. Southern Nursery Assn. Res. Conf. 52:102-108.

Currey, C.J., D.M. Camberato, A.P. Torres, and R.G. Lopez. 2010. Plant growth retardant drench efficacy is not affected by substrate containing parboiled rice hulls. HortTechnology 20:863-866.

Currey, C.J., K.J. Walters, and K.G. McCabe. 2016 Plant growth regulator drenches affect lantana cultivars differently. HortTechnology 26:20-25.

Currey, C.J. and N.J. Flax. 2015. Ethephon foliar sprays prevent premature flowering of tissue culture-propagated streptocarpus hybrids. HortTechnology 25:635-638.
Currey, C.J. and R.G. Lopez. 2011. Early flurprimidol drench applications suppress final height of four poinsettia cultivars. HortTechnology 21 : $35-40$.

Currey, C.J., R.G. Lopez, B.A. Krug, I. McCall, and B.E. Whipker. 2012. Substrate drenches containing flurprimidol suppress height of 'Nellie White' easter lilies. HortTechnology 22:164-168.

Dole, J.M. and H.F. Wilkins. 2005. Floriculture: Principles and species. 2nd ed. Pearson Prentice Hall, Upper Saddle River, NJ.

Erwin, J.E. and R.D. Heins. 1995. Thermomorphogenic responses in stem and leaf development. HortScience 30:940-949.

Fair, B.A., B. Whipker, I. McCall, and W. Buhler. 2012. Height control of 'Hot Lips' hybrid sage to flurprimidol substrate drench. HortTechnology 22:539-541.

Gent, M.P.N. and R.J. McAvoy. 2000. Plant growth retardants in ornamental horticulture, p. 89-146. In: A.S. Basara (ed.). Plant growth regulators in agriculture and horticulture: Their role and commercial uses. Food Products Press, Binghamton, NY.

Glady, J.E., N.S. Lang, and E.S. Runkle. 2007. Effect of ethephon on stock plant management of Coreopsis verticillata, Dianthus caryophyllus, and Veronica longifolia. HortScience 42:16161621.

Haver, D.L. and U.K. Schuch. 1996. Production and postproduction performance of new guinea Impatiens cultivars grown with controlled-release fertilizer and no leaching. J. Amer. Soc. Hort. Sci. 121:820-825.
Hayashi, T., R.D. Heins, A.C. Cameron, and W.H. Carlson. 2001. Ethephon influences flowering, height, and branching of several herbaceous perennials. Sci. Hort. 91:305-323.

Miller, W.B., N.S. Mattson, X. Xie, D. Xiu, C.J. Currey, K.L. Clemens, R.G. Lopez, M. Olrich, and E.S. Runkle. 2012. Ethephon substrate drenches inhibit stem extension of floriculture crops. HortScience 47:1312-1319.

Moe, R. 1980. The use of ethephon for control of plant height in daffodils and tulips. Acta Hort. 109:197-204.

Rademacher, W. 2000. Growth retardants: Effects on gibberellin biosynthesis and other metabolic pathways. Annu. Rev. Plant Physiol. Plant Mol. Biol. 51:501-531.

Runkle, E. 2013. Using the PGRs Collate and Florel. Greenhouse Product News 23(6):58.

U.S. Department of Agriculture. 2016. Floriculture crops 2015 summary. Nat. Agr. Sta. Service, Washington DC. 27 July 2016. <http://usda. mannlib.cornell.edu/usda/current/FlorCrop/ FlorCrop-04-26-2016.pdf>.

Vernieri, P., G. Incrocci, F. Togononi, and G. Serra. 2003. Effect of cultivar, timing, growth retardants, potting type on potted sunflowers production. Acta Hort. 614:313-318.

Wees, D. 1993. Effects of ethephon and ancymidol on plant height and flower abortion of easter lily. Can. J. Plant Sci. 73:879-883.

Whipker, B.E., I. McCall, and J. Latimer. 2011. Growth regulators, p. 95-105. In: J. Nau (ed.). Ball redbook, Vol. 2. Crop production. 18th ed. Ball Publ., West Chicago, IL. 